\title{
Influência da configuração urbana na geração fotovoltaica com sistemas integrados às fachadas
}

\author{
Influence of the urban layout on the energy generation of \\ building integrated photovoltaic systems
}

\section{Laura Rendón Gaviria \\ Fernando Oscar Ruttkay Pereira Martín Ordenes Mizgier}

\section{Resumo

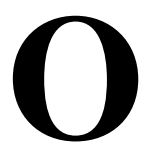

processo de adensamento das cidades e o aumento no consumo de energia fazem dos centros urbanos um dos setores de maior impacto no balanço energético mundial, sendo responsável por $70 \%$ do consumo total e energia. A geração de energia fotovoltaica apresenta uma grande vantagem, visto que geração e consumo podem ter coincidência espacial e temporal. Além disso, no caso das grandes cidades, as edificações apresentam formatos principalmente verticais e existe escassez de áreas horizontais disponíveis para a instalação de painéis fotovoltaicos. O objetivo do presente trabalho é, então, avaliar o potencial de aplicação de painéis fotovoltaicos em fachadas de edificações dentro de diferentes configurações urbanas, considerando o impacto do entorno construído. Foram analisados diferentes modelos, variando parâmetros arquitetônicos e urbanos, por meio de simulações dinâmicas no plug-in Diva, dentro do programa Rhinoceros. Estabeleceu-se para cada configuração urbana o potencial de geração fotovoltaica das superfícies verticais opacas e, finalmente, foram identificados os modelos uniformes como aqueles que apresentam a melhor relação entre demanda e geração. Os resultados deste estudo indicam que a determinação da eficiência dos modelos depende em grande medida da disponibilidade de superfícies para a geração FV (fachadas ou fachadas e cobertura). Comprovou-se um comportamento similar entre os modelos uniformes e mistos, e um decréscimo no desempenho dos cenários miolo de quadra, devido à reduzida área de cobertura, embora o vazio central ofereça altos níveis de acessibilidade solar nas fachadas.

Palavras-chave: Irradiação solar. Potencial fotovoltaico. Sistemas integrados. Contexto urbano. Relação geração/consumo.

\section{Abstract}

The urban densification process and increasing energy consumption make the urban environment one of the sectors with the highest impact on the world's energy balance, answering for $70 \%$ of the total energy consumption. The great advantage of photovoltaic $(P V)$ energy conversion is the possibility of spatial and temporal coincidence of generation and consumption. In large cities, buildings present mainly vertical formats and there are not enough horizontal areas available for PV installation. The aim of this study is to assess the application potential of $P V$ in building facades within different urban contexts, considering the impact of the surrounding built environment on solar radiation availability.

Different occupation models were analysed, with varying architectural and urban parameters, through dynamic simulations using the plug-in Diva, for Rhinoceros. The PV conversion potential for each non-glass vertical surface was established and, finally, the Uniform models were identified as the most efficient ones. The results of the study indicate that the efficiency of the model is highly dependent on the type of surface available for the generation of $P V$ (façades or façades and roofs). A similar behaviour was observed in Uniform and Mixed Models, as well as a decrease in the performance of Cloister Model due to a reduced roof area, even with the high daylight accessibility in the facades of the cloister.

Recebido em 21/06/13 Aceito em 12/07/13
Keywords: Solar radiation. Photovoltaic generation. Integrated Systems. Urban environment. Generation/consumption ratio. 


\section{Introdução}

Os processos de urbanização dos territórios vêm ocorrendo com uma tendência crescente e ocasionando significativos acréscimos nos consumos energéticos, simplesmente pelo aumento na quantidade de pessoas e equipamentos estabelecidos no mesmo território. Conforme Assis (2002), o adensamento dos núcleos urbanos pode ocasionar reduções importantes nos desempenhos dos edifícios e, consequentemente, aumentos sensíveis no consumo de energia referente à iluminação artificial e condicionamento do ar das edificações.

De acordo com Ruano (1999), os centros urbanos são responsáveis por $70 \%$ do total da energia consumida no mundo. Por sua vez, as edificações comerciais e residenciais computam 52\% da energia elétrica consumida na América Latina (INTERNATIONAL..., 2011). Fica claro, então, o impacto significativo que têm as cidades, como unidades e como sistemas, sobre o consumo final da energia e, consequentemente, o potencial existente para diminuição de forma significativa de grande parcela de energia que é consumida pelas edificações residenciais e comerciais, considerando que pequenas diminuições no consumo podem representar grandes economias energéticas.

O consumo final de energia no planeta tem apresentado uma tendência de crescimento de aproximadamente $2 \%$ por ano, porém a parcela dessa energia que é gerada por fontes renováveis só experimentou um crescimento de 1,7\% de 1973 até 2009 (INTERNATIONAL..., 2011).

No caso do Brasil, por se tratar de um país tropical com alta disponibilidade de irradiação solar, a geração de energia elétrica por meio do efeito fotovoltaico tem um grande potencial. Além de ser uma fonte renovável e praticamente inesgotável, a radiação solar, não poluente, é silenciosa e não causa efeitos negativos sobre o meio ambiente. Os centros urbanos apresentam as maiores densidades construídas, com a predominância de edificações de grande altura, com usos comerciais e residenciais; estas são responsáveis por $11 \%$ e $23 \%$ do total da energia elétrica consumida no Brasil respectivamente (LAMBERTS; PEREIRA; DUTRA, 1997). Nesses casos, devido à reduzida disponibilidade de superfícies horizontais para a instalação de painéis fotovoltaicos, urge a avaliação do potencial das superfícies verticais como local de instalação dos painéis.

À luz da Resolução Normativa $n^{\circ} 482$ (AGÊNCIA..., 2012), as edificações com instalação de sistemas fotovoltaicos têm a possibilidade de interligação à rede elétrica pública de forma a usufruir da energia quando a geração fotovoltaica não seja suficiente e injetá-la quando a geração supere o consumo. Por essa razão foram comparados os dados compilados de geração e consumo de um ano completo.

Dessa forma, o objetivo principal do presente trabalho é avaliar o potencial de geração de energia elétrica através de sistemas de painéis fotovoltaicos nas fachadas de edificações em função de diferentes configurações urbanas, considerando o impacto do entorno construído sobre a disponibilidade de irradiação solar no meio urbano.

\section{Revisão bibliográfica}

Dependendo de sua relação com a arquitetura, os sistemas fotovoltaicos podem-se dividir em BAPV (Building Applied Photovoltaics) e BIPV (Building Integrated Photovoltaics). Os primeiros são aqueles sistemas fixados ao envelope da edificação, mas que não fazem parte funcional dele, enquanto os BIPV são aqueles módulos FV que fazem parte da estrutura ou do envelope da edificação e são integrados a ela também esteticamente (JELLE; BREIVIK; RØKENES, 2012).

No universo dos estudos voltados para a integração de sistemas fotovoltaicos em edificações nos centros urbanos, a grande parte tem focado na consideração do nível de sombreamento das coberturas das edificações (LOULAS et al., 2012; HOFIERKA; KAŇUK, 2009) para assim calcular a área, ângulo de inclinação e tipo de painel ótimo para garantir a máxima eficiência do sistema de conversão. Embora alguns estudos (CRONEMBERGER; CAAMAÑO-MARTÍN; SÁNCHEZ, 2012; DI VINCENZO; KESTEN; INFIELD, 2010; VARTIAINEN, 2001; ORDENES et al., 2007; HWANGM; KANG; KIM, 2012) indiquem as superfícies verticais como áreas com bom potencial para a geração de energia fotovoltaica no meio urbano, ainda não se tem quantificado esse potencial.

A grande maioria dos estudos realizados sobre acesso solar nos ambientes urbanos avalia as edificações dentro do contexto edificado. Entretanto, é realizada a análise do comportamento de uma única edificação, e não do desempenho de um grupo de edificações. Isso representa uma limitação, visto que fazer a avaliação isolada de uma edificação, levando em consideração a influência do contexto, vai refletir na recomendação de parâmetros arquitetônicos e 
urbanos para as edificações vizinhas sem analisar o desempenho delas (LITTLEFAIR, 1998).

Tregenza (1998) e Ng (2001) desenvolveram distintos métodos para estimar o ingresso de luz natural em ambientes urbanos altamente adensados, demonstrando a grande relevância das alturas das edificações na caracterização do fenômeno no meio urbano. Na mesma linha, outros autores, como Ng e Wong (2004), Leder e Pereira (2009), Li et al. (2006) e Sánchez et al. (2005), avaliaram a disponibilidade de luz natural ou radiação solar nos envelopes de edificações urbanas para indicar os parâmetros construtivos mais influentes.

Todavia, vários estudos levam em consideração a integração de sistemas FV nas superfícies verticais de edificações, seja diretamente nas fachadas ou integrados a elementos de sombreamento (VARTIAINEN, 2001; ORDENES et al., 2007; HWANGM; KANG; KIM, 2012), com o objetivo de calcular o potencial de geração energética da edificação. Entretanto, as edificações foram avaliadas sem a influência de sombreamento ou contexto edificado algum.

A quantificação da disponibilidade solar nos contextos urbanos requer estudos mais detalhados por causa do fato de o sombreamento ser um fenômeno dinâmico. Como decorrência, é necessário o desenvolvimento de métodos que apoiem engenheiros e arquitetos na concepção de sistemas fotovoltaicos eficientes integrados às edificações (ZOMER, 2010).

Contudo, os estudos que propõem a instalação de sistemas fotovoltaicos nas fachadas não avaliam a influência do sombreamento. Similarmente, aqueles estudos que analisam a disponibilidade solar nas superfícies verticais de prédios dentro de contextos urbanos não quantificam o potencial de geração energética por meio da instalação de sistemas FV. Visando preencher essa lacuna na bibliografia, surge, então, o objetivo principal do presente trabalho: encontrar o potencial que fachadas de edificações dentro de contextos urbanos possuem para a geração de energia fotovoltaica, resultando na identificação dos parâmetros de construção urbana mais influentes na disponibilidade de radiação solar nas superfícies dessas edificações.

\section{Metodologia}

A pesquisa será um estudo exploratório que visa avaliar as fachadas de diferentes conformações urbanas para identificar seu potencial de geração de energia por meio de painéis FV. Foram escolhidos os parâmetros usados na construção dos cenários urbanos e, com o auxílio do programa Rhinoceros, foram produzidos os modelos computacionais. A partir da simulação computacional de nove modelos por meio do plug in Diva, foram obtidos os dados de disponibilidade de radiação solar que têm as superfícies das edificações. Finalmente, foram recalculadas as áreas disponíveis para instalação FV nos nove cenários urbanos, depois de lhes atribuir o nível PAF (percentual de abertura de fachada), para realizar novamente os cálculos de disponibilidade de radiação solar nas superfícies das edificações. Com esses dados e o sistema FV designado para cada edificação, foi possível calcular a contribuição na geração de energia de cada unidade e do contexto urbano completo. Finalmente, foram realizadas comparações entre a demanda de energia de cada modelo urbano e, através dessa comparação, foram classificadas segundo a porcentagem da demanda atendida pela geração FV em cada um deles.

\section{Definição dos cenários urbanos}

Os modelos dos cenários urbanos foram construídos com o intuito de avaliar a influência dos principais parâmetros utilizados no controle da ocupação urbana.

Estão listadas a seguir as variáveis utilizadas para a geração dos diferentes cenários:

(a) área de análise dos cenários. Foi estabelecida uma área quadrada de $100 \mathrm{~m}$ x $100 \mathrm{~m}$, representando uma porção de cidade onde foram inseridas 25 edificações uniformemente espaçadas entre si;

(b) área construída. Esse parâmetro também foi mantido constante e estabelecido em 35965,62 $\mathrm{m}^{2}$. Refere-se à área total construída de cada modelo;

(c) taxa de ocupação (T.O). É definida como a razão entre a projeção horizontal da área construída e a área total do terreno/lote, expressa percentualmente. Foram definidas três porcentagens diferentes, $40 \%, 30 \%$ e $20 \%$. Cabe salientar que, mantendo a área construída constante, a variação na taxa de ocupação influencia na variação dos afastamentos entre edificações e a altura delas, duas variáveis com grande influência na disponibilidade de radiação solar nos contextos urbanos, segundo Pereira et al. (2008), Ng e Wong (2004) e Di Vincenzo, Kesten e Infield (2010). Estabeleceram-se então três modelos de planta urbana, conforme os três valores da taxa de ocupação;

(d) tipologia urbana. Foram definidas três tipologias urbanas a serem aplicadas nas três 
plantas urbanas base geradas: uniforme, mista e miolo de quadra (Figura 1); e

(e) largura das ruas. Uma vez que cada agrupamento de 25 edificações pode representar uma quadra na malha urbana, os modelos foram construídos replicando-se a quadra base nove vezes e avaliando-se só a quadra central, garantindo, assim, uma aproximação de vizinhança de um contexto urbano. As separações entre as quadras, representando as ruas, foram definidas como vias subcoletoras de $16 \mathrm{~m}$, obedecendo às especificações do Plano Diretor de Florianópolis (PREFEITURA...; INSTITUTO..., 1998).

Os gabaritos (alturas dos blocos) e os afastamentos entre blocos foram determinados em função da área construída e da área de projeção de cada edificação.

Com essas escolhas e a combinação das tipologias e taxas de ocupação, foram produzidos nove cenários urbanos, conforme a Figura 2. As características principais dos modelos são ainda apresentadas na Tabela 1. Os níveis das refletâncias das superfícies foram tomados da pesquisa de Cherqui et al. (2005).

\section{Ferramentas de análise}

\section{Programa Rhinoceros 4.0}

Rhinoceros é uma ferramenta de modelagem 3D desenvolvida por Robert McNeel \& Associates, que permite a representação de uma grande quantidade de elementos em diversas escalas. Trata-se, principalmente, de um modelador de superfícies tipo NURBS (non-uniform rational $B$ splines), que são representações matemáticas de geometrias 3D que descrevem de maneira precisa elementos de qualquer formato. Adicionalmente, é possível editar a geometria, orientação, tipologia e materiais dos elementos, para exportá-los a outros programas ou para incluí-los em simulações dentro de algum dos diversos plug-ins compatíveis com o programa (ROBERT..., 2008).

Figura 1 - Tipologias urbanas selecionadas para a criação dos modelos
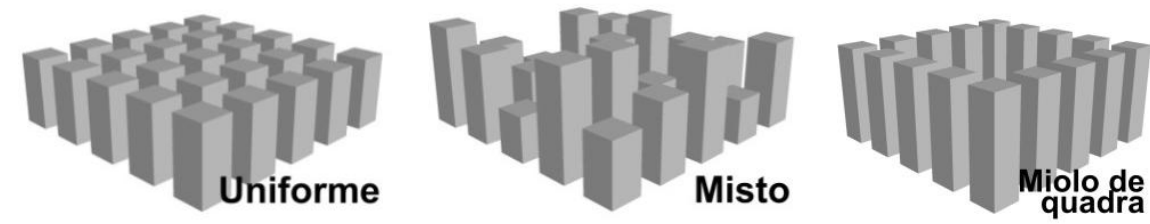

Figura 2 - Caracterização dos principais parâmetros arquitetônicos dos modelos

\begin{tabular}{|c|c|c|c|c|c|c|}
\hline $\begin{array}{l}\text { Taxa } \\
\text { de ocupação }\end{array}$ & Modelo & $\begin{array}{l}\text { Dimensões [d] } \\
\text { da Edificação }\end{array}$ & $\begin{array}{l}\text { Número } \\
\text { de andares }\end{array}$ & $\begin{array}{c}\text { Afastamento } \\
\text { entre edificaçoes } \\
\text { [a] }\end{array}$ & $\begin{array}{c}\text { Largura } \\
\text { das ruas }\end{array}$ & Código \\
\hline \multirow[t]{3}{*}{$20 \%$} & uniforme & \multirow{3}{*}{$\begin{array}{c}8,94 \mathrm{~m} \\
x \\
8,94 \mathrm{~m}\end{array}$} & 18 & \multirow{3}{*}{ 2. $13,38 \mathrm{~m}$} & \multirow{3}{*}{$16 \mathrm{~m}$} & 20_uni_t \\
\hline & misto & & $14-16-18-20-22$ & & & 20_mis_T \\
\hline & $\underset{\text { quadra }}{\text { miolo de }}$ & & $0-28$ & & & 20_mio_T \\
\hline \multirow{3}{*}{$30 \%$} & uniforme & \multirow{3}{*}{$\begin{array}{c}10,95 \mathrm{~m} \\
x \\
10,95 \mathrm{~m}\end{array}$} & 12 & \multirow{3}{*}{ 11,31m } & \multirow{3}{*}{$16 m$} & 30_uni_t \\
\hline & misto & & $8-10-12-14-16$ & & & 30_mis_T \\
\hline & $\underset{\text { quadra }}{\text { miolo de }}$ & & $0-19$ & & & 30_mio_T \\
\hline \multirow[t]{3}{*}{$40 \%$} & uniforme & \multirow{3}{*}{$\begin{array}{c}12,64 m \\
x \\
12,64 m\end{array}$} & 9 & \multirow{3}{*}{$9,20 \mathrm{~m}$} & \multirow{3}{*}{$16 \mathrm{~m}$} & 40_uni_T \\
\hline & misto & & $5-7-9-11-13$ & & & 40_mis_T \\
\hline & $\underset{\text { quadra }}{\operatorname{miolo} \text { de }}$ & & $0-14$ & & & 40_mio_T \\
\hline
\end{tabular}

Tabela 1 - Características dos modelos e inputs para a simulação

\begin{tabular}{c|c|c|c}
\hline Refletância edificações & Refletância piso & Consumo padrão & Consumo total \\
\hline $35 \%$ & $10 \%$ & $89 \mathrm{kWh} / \mathrm{m}^{2} / \mathrm{ano}$ & $3.200 .940 \mathrm{kWh} / \mathrm{ano}$ \\
\hline
\end{tabular}

10 Gaviria, L. R.; Pereira, F. O. R.; Mizgier, M. O. 
Os modelos tridimensionais foram confeccionados por meio de superfícies planas, que representam a volumetria das edificações dentro dos cenários urbanos. O programa permite a entrada das medidas exatas manualmente, garantindo um alto nível de precisão. Contudo, não será aplicado nenhum material às superfícies, visto que esse é um dos parâmetros de entrada no passo da simulação.

\section{Plug-in DIVA 2.0}

O plug-in foi desenvolvido por Jakubiec e Reinhard (2011), baseado em algoritmos de motores de simulação como Radiance, Daysim e EnergyPlus. O programa trabalha sob a interface do programa Rhinoceros, possibilitando a manipulação gráfica dos modelos e a conferência dos inputs diretamente na tela.

Foi utilizado um arquivo climático de base horária TRY (Test Reference Year), que o possibilita a realização de simulações dinâmicas. O plug-in realiza a avaliação sobre uma série de nodos, localizados nas superfícies a serem analisadas. A densidade dos nodos em cada superfície determina, então, o nível de detalhe dos resultados (JAKUBIEC; REINHART, 2011; SOLEMMA, 2013).

Foram obtidos os resultados da radiação solar disponível nas superfícies da envoltória das edificações, dada em $\mathrm{kWh} / \mathrm{m}^{2}$.

\section{Simulações da disponibilidade de radiação solar}

A partir da construção dos nove modelos urbanos base no programa Rhinoceros, foram feitas simulações de disponibilidade de radiação solar nas superfícies das edificações da quadra central, por meio do plug-in DIVA. Os resultados das simulações, expressados em $\mathrm{kWh} / \mathrm{m}^{2} / \mathrm{ano}$, foram armazenados em tabelas eletrônicas, de forma a possibilitar o cálculo da quantidade de irradiação solar disponível em cada cenário urbano, como também a contribuição de cada edificação.

\section{Criação das faixas de disponibilidade solar}

As superfícies de cada edificação foram divididas em três faixas de disponibilidade de radiação solar (DRS). As faixas foram estabelecidas a partir dos níveis mínimos de radiação para aproveitamento FV recomendados na Europa. Segundo Cronemberger, Caamaño-Martín e Sánchez (2012), o limite mínimo europeu é recomendado como $60 \%$ do valor máximo.

Foi estabelecido o limite mínimo de radiação solar disponível aproveitável para conversão FV nas superfícies de edificações inseridas em contextos urbanos. Visando gerar uma comparação equilibrada, levou-se em consideração que se trata de uma situação restrita, devido à grande quantidade de obstruções que apresentam as superfícies. Foi tomada como base a recomendação europeia de $60 \%$ do total disponível, porém, pelas razões explicadas acima, e levando em consideração que o nível máximo de radiação disponível nos modelos estudados foi $1.376 \mathrm{kWh} / \mathrm{m}^{2}$, adotou-se uma redução à recomendação. Adotou-se, então, 40\% como limite mínimo de radiação solar disponível para aproveitamento fotovoltaico do presente trabalho.

Na Tabela 2 são apresentados os valores para alguns países europeus e os valores calculados para Florianópolis.

Levando-se em consideração a grande quantidade de obstruções que apresentam as superfícies verticais nos diferentes cenários, adotou-se como limite mínimo de radiação solar disponível para aproveitamento fotovoltaico de $40 \%$ do máximo total disponível. Por sua parte, adotou-se aproximadamente $60 \%$ como o limite para a criação da faixa de radiação solar média. Com base nisso, a definição das faixas DRS foi realizada como indica a Tabela 3 .

Tabela 2 - Limite mínimo de disponibilidade de irradiação solar ( $\mathrm{kWh} / \mathrm{m}^{2}$.ano) para aproveitamento FV em superfícies verticais em ambientes urbanos na Europa e em Florianópolis

\begin{tabular}{|c|c|c|c|c|c|c|}
\hline & \multicolumn{3}{|c|}{ Europa** } & \multirow{2}{*}{\multicolumn{3}{|c|}{ Florianópolis }} \\
\hline & Alemanha & Espanha & França & & & \\
\hline Níveis máximos & 1.250 & 1.850 & 1.650 & \multicolumn{3}{|c|}{$1.376,85^{*}$} \\
\hline Nível mínimo & \multicolumn{3}{|c|}{$60 \%$} & & & \\
\hline recomendado (Europa) & 750 & 1.110 & 990 & & & \\
\hline \multirow{2}{*}{$\begin{array}{l}\text { Nível mínimo calculado } \\
\text { (Florianópolis) }\end{array}$} & & & & $40 \%$ & $50 \%$ & $60 \%$ \\
\hline & & & & 550 & 688 & 826 \\
\hline
\end{tabular}

Nota: * Irradiação solar máxima disponível nos modelos urbanos do presente estudo $\left(\mathrm{kWh} / \mathrm{m}^{2}\right.$.ano).

** Tomado de Cronemberger, Caamaño-Martín e Sánchez (2012). 
Tabela 3 - Definição das faixas DRS segundo a disponibilidade de irradiação solar

\begin{tabular}{c|c}
\hline Faixa DRS & Média da disponibilidade de radiação solar \\
\hline Baixa & $0-550 \mathrm{kWh} / \mathrm{m}^{2} /$ ano \\
Média & $551-800 \mathrm{kWh} / \mathrm{m}^{2} /$ ano \\
Alta & $>800 \mathrm{kWh} / \mathrm{m}^{2} /$ ano \\
\hline
\end{tabular}

\section{Definição do PAF}

Para avaliar o real potencial que tem determinada fachada para receber módulos fotovoltaicos opacos integrados à fachada, é necessário considerar o espaço disponível, descontando-se a área das aberturas.

No cálculo inicial do potencial de geração fotovoltaica de uma fachada, não serão levadas em consideração as aberturas nas edificações. Dessa forma, é possível avaliar a redução do potencial de geração para cada modelo, considerando a redução da área de aplicação dos painéis devido à existência das aberturas.

Para a definição dos valores do percentual de abertura de fachada (PAF) a serem aplicados nas fachadas (Tabela 4), considera-se as três faixas de disponibilidade solar. As áreas das edificações que se situam na faixa DRS alta devem aproveitar o máximo possível de área conversora, tendo, então, sua área envidraçada limitada, $\mathrm{PAF}=25 \%$. Nas porções com DRS média, será atribuído um PAF de $50 \%$. Finalmente, as áreas pertencentes à faixa DRS baixa contarão com um PAF de $75 \%$, levando em consideração que, além do baixo potencial de geração fotovoltaica, os ambientes associados apresentarão baixos níveis de iluminação natural, o que pode ser parcialmente resolvido com aberturas maiores.

\section{Escolha das características do sistema FV}

Existe no mercado uma grande variedade de sistemas fotovoltaicos, que variam em custo, eficiência, aparência e materiais de fabricação. Com o objetivo de escolher um sistema FV para realizar os cálculos de geração de energia, foi realizada uma revisão das ofertas atuais dos principais fabricantes de sistemas de conversão solar fotovoltaica do mercado. Finalmente, foi escolhido um módulo de silício multicristalino, do fabricante Yingli Solar, que conta com características físicas e de eficiência conforme a Tabela 5. A escolha visou adotar características médias entre os diferentes sistemas disponíveis no mercado, que não fosse nem o sistema mais eficiente nem com a eficiência mais baixa.

O rendimento do sistema escolhido é resultado de experimentos de laboratório realizados sob condições ótimas (Standard Test Conditions,
STC), que decorrem da suposição de níveis de eficiência diferentes aos que seriam alcançados no funcionamento real do sistema.

\section{Cálculo da geração de energia}

A área de superfície disponível para a instalação dos painéis foi calculada para cada um dos 9 cenários urbanos, já com os valores de PAF correspondentes, dependendo da faixa DRS na qual as porções das fachadas foram classificadas.

A partir dos dados de disponibilidade de irradiação solar, e levando em consideração o sistema FV adotado, foi calculada a quantidade de energia produzida por cada bloco no período de um ano. Por fim, as gerações energéticas das 25 edificações de cada contexto foram somadas, resultando na quantidade total de energia gerada em cada modelo urbano. O cálculo foi realizado por meio da seguinte Equação 1:

$\mathrm{E}=\mathrm{G}$ X r X Pcc

Eq. 1

Onde:

E é a energia gerada, em kWh/ano;

$\mathrm{G}$ é a irradiação diária recebida no plano do painel fotovoltaico $\left(\mathrm{kWh} / \mathrm{m}^{2}\right)$, dividida pela irradiância de referência de $1 \mathrm{~kW} / \mathrm{m}^{2}$, expressa em número de horas (h);

r é o rendimento ou coeficiente de desempenho do sistema fotovoltaico como um todo. Foi adotado 0,80 , por ser um valor comumente recomendado na literatura (RÜTHER, 2004; ZOMER, 2010); e

Pcc é a potência do sistema em corrente contínua $(\mathrm{kW})$.

A faixa DRS baixa não foi levada em consideração como superfície aproveitável para instalação de painéis $\mathrm{FV}$, visto que os níveis de irradiação são inferiores ao limite calculado como mínimo recomendado para o caso de Florianópolis. Tratase de uma situação pouco favorável, dada a grande quantidade de painéis requeridos, considerando a reduzida geração. A Tabela 6 mostra a comparação da média de geração FV por metro quadrado nas três faixas DRS. Fica evidente a grande diferença entre a geração da faixa baixa e as outras duas faixas (alta e média), justificando num primeiro momento a não consideração daquela faixa para conversão fotovoltaica. 
Para possibilitar a comparação entre geração e consumo de energia elétrica, adotou-se um consumo constante ao longo do período, baseado no estudo de Santana (2006). Conforme a autora, para a tipologia predominante de edificações comerciais em Florianópolis, pode-se estabelecer um consumo de $89 \mathrm{kWh} / \mathrm{m}^{2} /$ ano.

\section{Resultados}

Nesta seção serão apresentados os principais resultados encontrados no desenvolvimento da metodologia proposta. Inicialmente, foi indicada a distribuição das áreas nas diferentes faixas DRS. Em seguida foram calculadas as áreas das edificações disponíveis para conversão FV, após diminuir a porcentagem de PAF. Finalmente, foi calculada a geração energética de cada modelo e realizada a comparação de geração e consumo de forma a encontrar o nível de eficiência de cada cenário.

\section{Divisão das Faixas de Disponibilidade de Radiação Solar}

Foram calculadas as áreas de cada modelo pertencentes a cada faixa DRS. A Figura 3 apresenta a comparação das áreas dos modelos e a subdivisão nas diferentes faixas.

Tabela 4 - Atribuição dos valores de PAF às faixas DRS

\begin{tabular}{c|c}
\hline Faixa DRS & PAF \\
\hline Baixa & $75 \%$ \\
Média & $50 \%$ \\
Alta & $25 \%$ \\
\hline
\end{tabular}

Tabela 5 - Características do módulo solar fotovoltaico adotado

\begin{tabular}{l|c|c|c|c|c}
\hline Fabricante & Modelo & Tecnologia & Eficiência & $\begin{array}{c}\text { Potência } \\
\text { nominal }\end{array}$ & $\begin{array}{c}\text { Dimensões } \\
{[\mathbf{m m}]}\end{array}$ \\
\hline Yingli Solar & YL 180P-23b & $\begin{array}{c}\text { Silício } \\
\text { multicristalino }\end{array}$ & $13,9 \%$ & $180 \mathrm{~W}$ & $1.310 * 990$ \\
\hline
\end{tabular}

Tabela 6 - Geração FV média de um metro quadrado de superfície nas diferentes Faixas DRS

\begin{tabular}{c|c}
\hline Faixa DRS & $\mathbf{k W h} / \mathbf{m}^{\mathbf{2}} \mathbf{a n o}$ \\
\hline Baixa & 39,6 \\
Média & 97,2 \\
Alta & 156,6 \\
\hline
\end{tabular}

Figura 3 - Divisão das áreas dos modelos nas diferentes faixas DRS

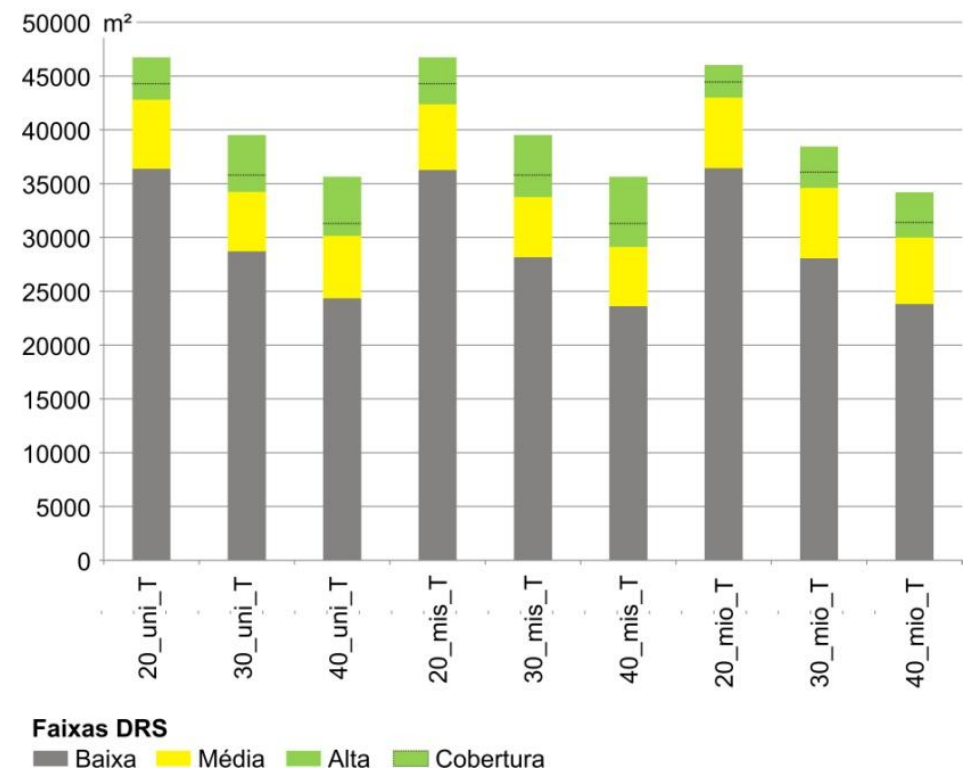


Conforme a Tabela 7 , nos modelos de TO de $20 \%$, em torno de $80 \%$ da área total das superfícies ficou classificada na faixa DRS baixa; nos modelos de TO de $30 \%$ esta porcentagem caiu para $72 \%$, e, finalmente, nos modelos com TO de $40 \%$, a faixa baixa representou até $69 \%$ da área total. O elevado nível de obstrução apresentado pelas fachadas em ambientes urbanos é provavelmente o aspecto responsável por esse efeito. Contudo, nos modelos de TO de $20 \%$, a faixa alta apresenta áreas similares nas fachadas e nas coberturas, visto que é um cenário com uma área de cobertura bastante reduzida. Embora a cobertura seja usualmente a superfície com maior irradiação solar, nesse caso, considerando a divisão das faixas, as fachadas apresentam áreas com bom potencial de conversão.

Cabe salientar que, embora os modelos com taxas de ocupação mais baixa apresentem maiores áreas disponíveis para a instalação de painéis FV e maiores afastamentos entre edificações, a relação entre a área total externa dos modelos uniformes e a área pertencente à faixa média (entre 551 e 800 $\mathrm{kWh} / \mathrm{m}^{2}$.ano) não é diretamente proporcional. É no modelo 30_uni_T em que se encontra a maior área na faixa média; já na faixa alta, é o modelo 20_uni_T que apresenta maior área, quando levadas em consideração as superfícies das fachadas e cobertura. Contudo, na somatória das áreas de fachadas e cobertura, o modelo com maior área na faixa alta é o 40_mis_T, o que pode ser explicado pelo tamanho maior das coberturas e as grandes áreas que não apresentam bloqueios por causa da variação dos gabaritos (Figura 4). No caso do modelo 40_mio_T, embora tenha a mesma área de cobertura em cada edificação, o número reduzido de edificações traz como decorrência um decréscimo importante na disponibilidade de radiação solar. Vale a pena salientar que, embora as condições de acessibilidade solar sejam bastante superiores nas fachadas dos modelos Miolo de Quadra, dado que no centro da quadra não se dispõem edificações, a diminuição no número de blocos e, então, na área de superfícies externas disponíveis para integração FV é um fator de maior peso na quantidade de radiação solar.

Tabela 7 - Divisão das áreas dos modelos nas diferentes faixas DRS ( $\left.\mathrm{m}^{2} \mathrm{e} \%\right)$



Figura 4 - Níveis de radiação solar disponível nas superfícies externas - Modelos Uniformes

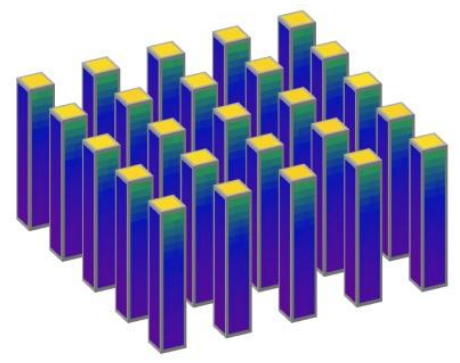

20_uni_T

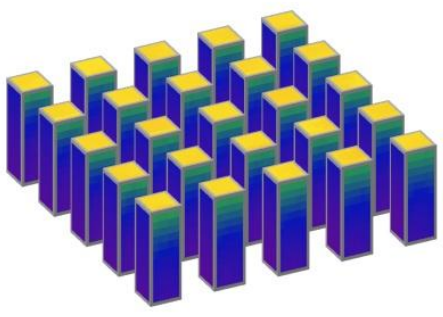

30_uni_T

Radiação $\mathrm{kWh} / \mathrm{m}^{2} /$ ano

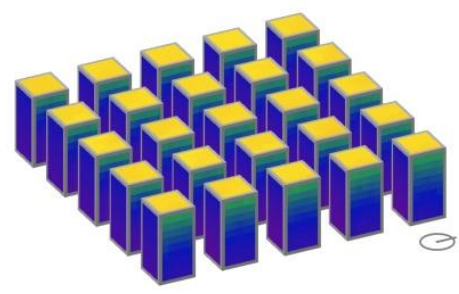

40_uni_T

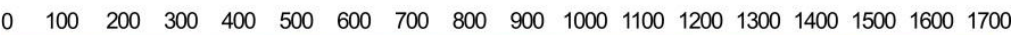

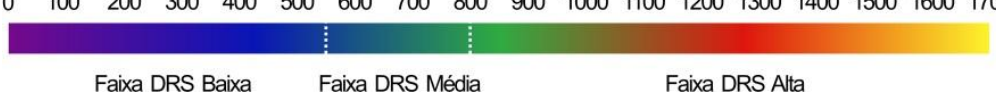

Faixa DRS Baixa Faixa DRS Média Faixa DRS Alta

14 Gaviria, L. R.; Pereira, F. O. R.; Mizgier, M. O. 
No caso dos modelos Mistos, pela aleatoriedade nas alturas das edificações, observam-se amplas áreas de fachada bastante expostas e outros prédios com níveis de radiação bastante restritos pelo sombreamento (Figura 5). Infere-se que a disponibilidade de radiação solar nas superfícies externas teria um comportamento menos previsível, porém é conferida uma compensação, visto que a quantidade total de irradiação solar é similar à recebida nos cenários Uniformes. Contudo, é evidente que nos cenários dessa tipologia, diferentemente do que acontece nos cenários Uniformes, os comportamentos dos prédios, quando avaliados isoladamente, são bastante heterogêneos. Essa diferença entre disponibilidade de radiação solar decorre da variação aleatória entre as alturas das edificações adjacentes.

Nessa mesma linha, é interessante apontar que, no caso dos cenários Miolo de Quadra, as edificações que apresentam melhores desempenhos não pertencem ao lado norte da quadra. Isso pode ser explicado pelo fato de que a maior quantidade de radiação (que provem do norte) tem mais fácil acesso aos blocos sul por meio do vazio da quadra. Similarmente, as edificações no leste e oeste recebem apreciáveis quantidades de radiação através do vazio (Figura 6). É interessante apontar, então, que são as fachadas voltadas para o interior do cenário as que contam com os melhores desempenhos.

Figura 5 - Níveis de radiação solar disponível nas superfícies externas - Modelos Mistos

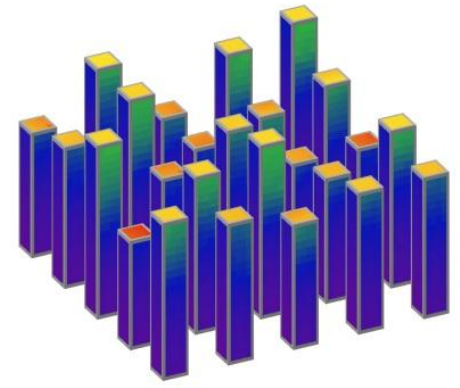

20_mis_T

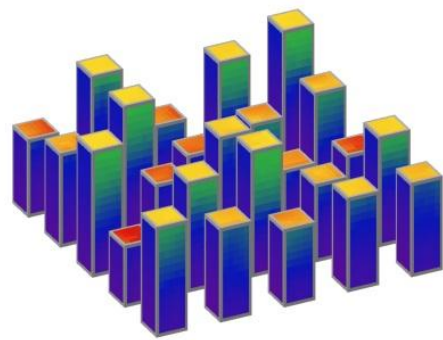

30_mis_T

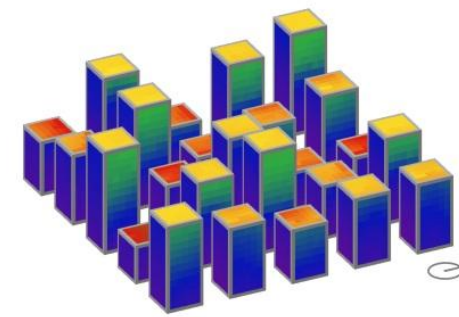

40_mis_T

$\mathrm{Wh} / \mathrm{m}^{2} / \mathrm{an}$ o

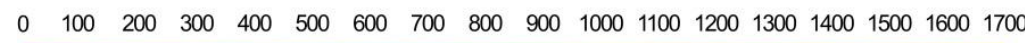

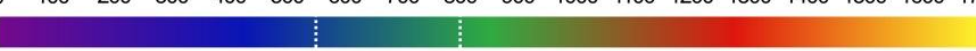

Faixa DRS Baixa $\quad$ Faixa DRS Média

Faixa DRS Alta

Figura 6 - Níveis de radiação solar disponível nas superfícies externas - Modelos Miolo de Quadra

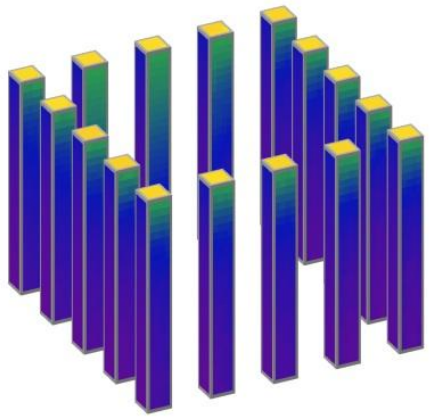

20_mio_T

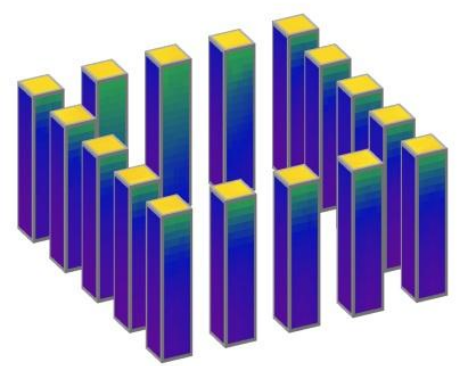

30_mio_T

Radiação $\mathrm{kWh} / \mathrm{m}^{2} /$ ano

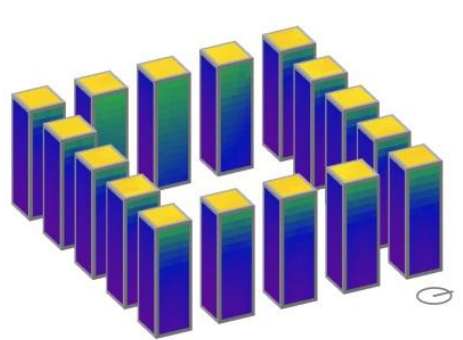

40_mio_T





\begin{tabular}{llll}
\hline Faixa DRS Baixa Faixa DRS Média & Faixa DRS Alta
\end{tabular} 


\section{Cálculo da área opaca disponível para instalação dos painéis}

Como já colocado anteriormente, para o cálculo da geração fotovoltaica é importante levar em consideração a presença aberturas, nas quais não é possível a instalação de painéis $\mathrm{FV}$.

Na Tabela 8 são apresentadas as áreas resultantes da redução do PAF em cada faixa DRS.

Depois do recálculo das áreas já sem o PAF correspondente a cada faixa, são os modelos Mistos os que têm maior disponibilidade de radiação solar na faixa alta das superfícies verticais, e esta apresenta uma relação diretamente proporcional ao aumento no TO. Já no caso dos modelos Miolo de Quadra e Uniformes, o cenário com TO de $30 \%$ é o que apresenta a maior quantidade de área correspondente à faixa alta.

\section{Cálculo da geração de energia elétrica}

Na Tabela 9 são apresentadas as quantidades de energia, expressas em $\mathrm{kWh} / \mathrm{m}^{2} /$ ano, que os modelos produzem anualmente em cada uma de suas faixas DRS.

Em todos os modelos, conforme a Taxa de Ocupação aumenta, a quantidade de energia gerada também aumenta, em especial devido às coberturas. Porém, o aumento na geração de energia não registra uma tendência linear como apresenta o aumento dos níveis de TO. Entretanto, quando é comparada a geração de energia só das fachadas, o aumento do TO provoca uma leve tendência de diminuição na produção de energia. Também é percebido que o aumento da Taxa de Ocupação não representa aumentos proporcionais na geração energética, mostrando uma curva de crescimento não linear e com tendência à estabilização. Essa condição deve acontecer em torno da condição de edificações térreas com TO teórica de $100 \%$, quando a geração seria máxima através das coberturas.

Tabela 8 - Áreas efetivas para instalação FV, diminuindo as áreas das aberturas segundo a faixa DRS $\left(\mathrm{m}^{2}\right)$

\begin{tabular}{c|c|c|c|c}
\hline & \multirow{2}{*}{ BAIXA } & \multirow{2}{*}{ MÉDIA } & \multicolumn{2}{|c}{ ALTA } \\
\cline { 4 - 5 } & & & Fac & Cob \\
\hline PAF & $75 \%$ & $50 \%$ & $25 \%$ & - \\
20_uni_T & 27.279 & 3.213 & 371 & 1.998 \\
30_uni_T & 21.528 & 2.765 & 391 & 3.120 \\
40_uni_T & 18.260 & 2.896 & 289 & 3.994 \\
20_mis_T & 27.196 & 3.054 & 478 & 1.998 \\
30_mis_T & 21.116 & 2.795 & 513 & 2.998 \\
40_mis_T & 17.702 & 2.757 & 544 & 3.994 \\
20_mio_T & 27.324 & 3.282 & 360 & 1.279 \\
30_mio_T & 21.044 & 3.269 & 369 & 1.918 \\
40_mio_T & 17.856 & 3.091 & 352 & 2.556 \\
\hline
\end{tabular}

Tabela 9 - Geração acumulada de energia FV de cada modelo para um ano completo (kWh/ano)

\begin{tabular}{l|c|c|c|c|c|c}
\hline & \multirow{2}{*}{ BAIXA } & \multirow{2}{*}{ MÉDIA } & \multicolumn{2}{|c|}{ ALTA } & \multirow{2}{*}{ TOTAL } & TOTAL \\
\cline { 4 - 6 } & & Fac & Cob & Fac & Fac + Cob \\
\hline 20_uni_T & - & 250.774 & 37.707 & 468.022 & 288.482 & 756.503 \\
30_uni_T & - & 216.133 & 39.200 & 709.985 & 255.333 & 965.319 \\
40_uni_T & - & 224.675 & 29.171 & 827.616 & 253.846 & 1.081 .462 \\
20_mis_T & - & 237.408 & 48.587 & 450.073 & 285.995 & 736.069 \\
30_mis_T & - & 219.025 & 52.447 & 671.062 & 271.473 & 942.534 \\
40_mis_T & - & 216.710 & 56.030 & 769.937 & 272.739 & 1.042 .677 \\
20_mio_T & - & 254.990 & 36.572 & 302.252 & 291.562 & 593.814 \\
30_mio_T & - & 253.291 & 37.553 & 452.174 & 290.844 & 743.018 \\
40_mio_T & - & 241.541 & 35.779 & 533.057 & 277.320 & 810.377 \\
\hline
\end{tabular}

16 Gaviria, L. R.; Pereira, F. O. R.; Mizgier, M. O. 


\section{Comparação entre geração e consumo}

A Figura 7 apresenta a razão entre geração e consumo de energia para os diferentes cenários. Isso significa que, quanto maior o valor, uma maior porção do consumo será atendida pela geração local. O cenário Uniforme apresenta razões levemente superiores que as do cenário Misto. Entretanto, o modelo Miolo de Quadra apresenta os desempenhos menos favorecidos. Um aspecto relevante é que a razão aumenta conforme a TO aumenta, quando se considera a energia gerada nas fachadas e nas coberturas. Porém, a tendência se inverte quando se computa apenas a energia gerada nas fachadas, com as fachadas apresentando uma participação na geração total de energia de até quase $10 \%$.

\section{Fichas-resumo da eficiência geral dos modelos}

Nesta seção são apresentadas as fichas-resumo da relação entre geração e consumo de cada cenário. $\mathrm{Na}$ parte esquerda da ficha apresenta-se a planta do modelo, ressaltando os cinco prédios que possuem as maiores contribuições na geração energética. No lado direito são apresentadas as contribuições de cada edificação na produção de energia total, possibilitando a comparação entre eles. Finalmente, na parte superior, encontram-se a identificação do modelo e a porcentagem do consumo total anual a que a geração FV consegue atender.
O modelo 40_uni_T obteve a maior eficiência entre todos os modelos, chegando perto de suprir $35 \%$ de sua demanda energética anual. Com 32,59\% segue o modelo 40_mis_T (Figura 7).

Pela Figura 8 pode-se perceber que, no caso dos cenários Uniformes com TO de 20\%, as edificações que contribuem em maior medida à geração de energia são edificações perimetrais, em sua maioria nas esquinas do modelo, porém só uma delas está orientada para o norte. É conferida uma influência importante da orientação e da localização dos prédios dentro do próprio cenário. Já nos modelos 30_uni_T e 40_uni_T o desempenho das edificações se vê influenciado pela redução do afastamento entre blocos, incorrendo em uma tendência clara de os prédios perimetrais orientados ao norte apresentarem melhores desempenhos.

As edificações que expõem os melhores desempenhos nos Modelos Mistos são, na maioria, aquelas que contam com gabaritos mais altos e, portanto, possuem maior exposição solar. Unicamente uma das edificações que apresenta um desempenho superior trata-se de um bloco mais baixo. Isso é explicado pela proximidade existente entre dois dos prédios mais altos do cenário, o que cria altos níveis de obstrução solar (Figura 9).

Já nos casos em que a geração é realizada unicamente nas fachadas, são os Modelos Miolo de Quadra os que apresentam as melhores relações entre geração e consumo, chegando até $9,32 \%$ no modelo de TO de $20 \%$ (Figura 10).

Figura 7 - Percentagem do consumo de cada cenário que é gerado pela produção FV

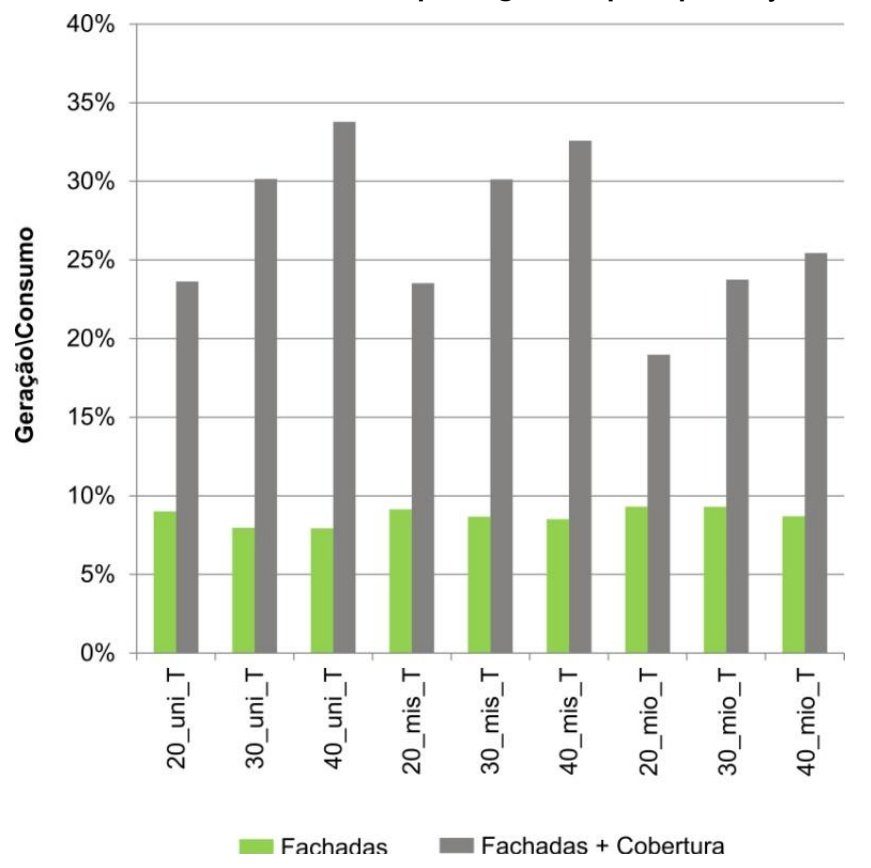


Figura 8 - Fichas-resumo do desempenho particular das edificações e da relação entre geração e consumo dos modelos Uniformes. Avaliação com e sem cobertura

\begin{tabular}{|c|c|c|c|c|c|c|c|c|c|c|c|}
\hline & a & b & & d & e & & & & $\%$ & \multicolumn{2}{|c|}{$\underset{\text { sem cobertura }}{20}$} \\
\hline 1 & 밈 & 口 & - & 口 & 口 & & a & b & c & d & e \\
\hline 2 & च & 口 & E & E & E & 1 & $4,28 \%$ & $3,96 \%$ & $4,11 \%$ & $3,97 \%$ & $4,07 \%$ \\
\hline 3 & 물 & $\square$ & च & [ & 口 & 2 & $4,12 \%$ & $3,95 \%$ & $3,87 \%$ & $4,02 \%$ & $3,94 \%$ \\
\hline & & & & & & 3 & $4,19 \%$ & $3,73 \%$ & $3,95 \%$ & $3,85 \%$ & $4,20 \%$ \\
\hline 4 & 口 & $\mathbf{\square}$ & 口 & 口 & 口 & 4 & $4,10 \%$ & $3,91 \%$ & $3,83 \%$ & $4,02 \%$ & $4,01 \%$ \\
\hline 5 & a & E & 口 & 口 & E & 5 & $4,20 \%$ & $3,73 \%$ & $3,73 \%$ & $4,06 \%$ & $4,20 \%$ \\
\hline & a & b & c & $d$ & & & & & & $\mathrm{com}$ & ni_t \\
\hline$T$ & 口 & घ & E & 口 & 口 & & a & b & c & d & e \\
\hline 2 & 口 & E & घ & [ & च & 1 & $4,10 \%$ & $3,99 \%$ & $4,07 \%$ & $3,98 \%$ & $4,04 \%$ \\
\hline 3 & 뭄 & 口 & D & 口 & & 2 & $4,04 \%$ & $3,98 \%$ & $3,94 \%$ & $3,99 \%$ & $3,97 \%$ \\
\hline & & & 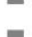 & & v & 3 & $4,08 \%$ & $3,91 \%$ & $3,98 \%$ & $3,94 \%$ & $4,06 \%$ \\
\hline 4 & 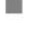 & 口 & 口 & 口 & 口 & 4 & $4,04 \%$ & $3,95 \%$ & $3,94 \%$ & $4,02 \%$ & $4,00 \%$ \\
\hline 5 & 口 & $\mathbf{E}$ & E & 口 & 口 & 5 & $4,07 \%$ & $3,91 \%$ & $3,92 \%$ & $4,02 \%$ & $4,08 \%$ \\
\hline
\end{tabular}

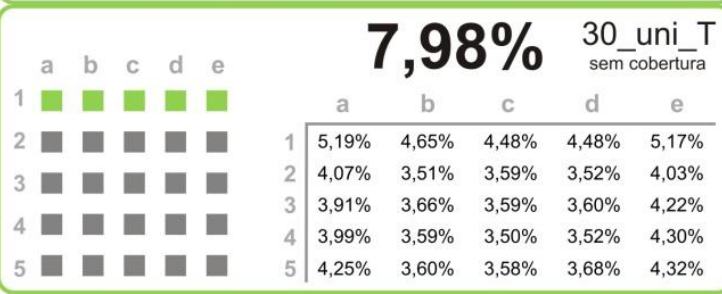

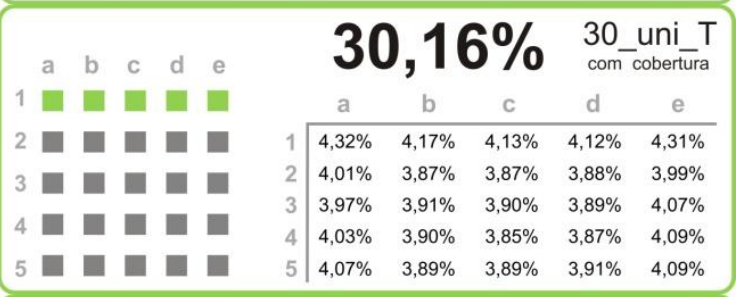

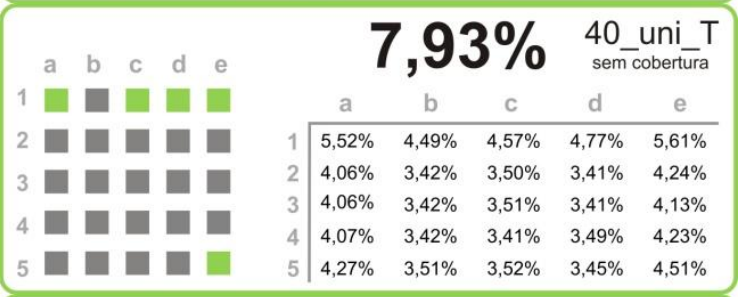

\begin{tabular}{|c|c|c|c|c|c|c|}
\hline 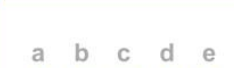 & & & & & $\begin{array}{l}40 \\
\text { com }\end{array}$ & uni_T \\
\hline $1 \square \square \square \square \square$ & & a & b & c & d & e \\
\hline$\square \square \square$ & 1 & $4,35 \%$ & $4,11 \%$ & $4,13 \%$ & $4,18 \%$ & $4,38 \%$ \\
\hline 3 & 2 & $4,04 \%$ & $3,87 \%$ & $3,87 \%$ & $3,84 \%$ & $4,06 \%$ \\
\hline$=$ & 3 & $4,02 \%$ & $3,86 \%$ & $3,88 \%$ & $3,86 \%$ & $4,04 \%$ \\
\hline $4 \square$ & 4 & $4,02 \%$ & $3,87 \%$ & $3,86 \%$ & $3,89 \%$ & $4,05 \%$ \\
\hline $5 \square \square \square \square \square$ & 5 & $4,05 \%$ & $3,89 \%$ & $3,90 \%$ & $3,86 \%$ & $4,11 \%$ \\
\hline
\end{tabular}


Figura 9 - Fichas-resumo do desempenho particular das edificações e da relação entre geração e consumo dos modelos Mistos. Avaliação com e sem cobertura

\begin{tabular}{|c|c|c|c|c|c|c|c|c|c|c|c|}
\hline & a & & & & e & & & & $1 \%$ & \multicolumn{2}{|c|}{$\underset{\text { sem cobertura }}{20 \text { mis_T }}$} \\
\hline 1 & 무 & घ & 口 & 口 & 口 & & a & b & c & d & e \\
\hline 2 & [ & घ & घ & 口 & घ & 1 & $7,60 \%$ & $3,93 \%$ & $0,29 \%$ & $7,09 \%$ & $3,03 \%$ \\
\hline 3 & $\mathbf{0}$ & 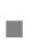 & 口 & 口 & 口 & 2 & $6,12 \%$ & $2,37 \%$ & $1,04 \%$ & $2,68 \%$ & $3,52 \%$ \\
\hline & & & & & & 3 & $1,05 \%$ & $1,17 \%$ & $6,49 \%$ & $7,73 \%$ & $2,16 \%$ \\
\hline 4 & 믐 & 口 & ㅁ & घ & 口 & 4 & $7,55 \%$ & $7,81 \%$ & $1,29 \%$ & $6,40 \%$ & $3,26 \%$ \\
\hline 5 & 口 & 口 & 口 & 口 & घ & 5 & $0,65 \%$ & $2,68 \%$ & $7,52 \%$ & $0,29 \%$ & $6,26 \%$ \\
\hline & a & b & c & to & & & & & & 20 & his_T \\
\hline 1 & 밈 & $\square$ & 口 & 口 & घ & & a & b & c & d & e \\
\hline 2 & 口 & 口 & 口 & 口 & 口 & 1 & $5,51 \%$ & $4,05 \%$ & $2,31 \%$ & $5,31 \%$ & $3,65 \%$ \\
\hline 3 & 口 & 口 & a & ㅁ & [ & 2 & $4,92 \%$ & $3,32 \%$ & $2,79 \%$ & $3,48 \%$ & $3,87 \%$ \\
\hline & & & & & & 3 & $2,76 \%$ & $2,77 \%$ & $5,04 \%$ & $5,55 \%$ & $3,25 \%$ \\
\hline 4 & in & 表 & E & a & a & 4 & $5,51 \%$ & $5,56 \%$ & $2,83 \%$ & $5,02 \%$ & $3,73 \%$ \\
\hline 5 & $\mathbf{a}$ & $\mathbf{\square}$ & 口 & 口 & घ & 5 & $2,62 \%$ & $3,51 \%$ & $5,46 \%$ & $2,24 \%$ & $4,95 \%$ \\
\hline
\end{tabular}

\begin{tabular}{|c|c|c|c|c|c|c|c|c|c|c|}
\hline a & b & c & & & & & & $0 \%$ & $\begin{array}{r}30 \\
\text { sen }\end{array}$ & nis_T \\
\hline 1 & 口 & [ & ⿷ & 口 & & a & b & c & d & e \\
\hline 2 & 口 & 口 & 口 & 口 & 1 & $9,26 \%$ & $4,03 \%$ & $0,14 \%$ & $8,31 \%$ & $2,90 \%$ \\
\hline 3 & $\square$ &  & F & $\square$ & 2 & $6,69 \%$ & $2,10 \%$ & $0,80 \%$ & $1,98 \%$ & $3,31 \%$ \\
\hline & & & & & 3 & $0,37 \%$ & $0,69 \%$ & $6,90 \%$ & $8,46 \%$ & $1,87 \%$ \\
\hline 4 & L & L & D & L & 4 & $8,39 \%$ & $8,22 \%$ & $0,14 \%$ & $5,90 \%$ & $2,95 \%$ \\
\hline 5 & $\mathbf{\square}$ & L & $\square$ & 口 & 5 & $0,00 \%$ & $1,58 \%$ & $8,42 \%$ & $0,00 \%$ & $6,57 \%$ \\
\hline
\end{tabular}

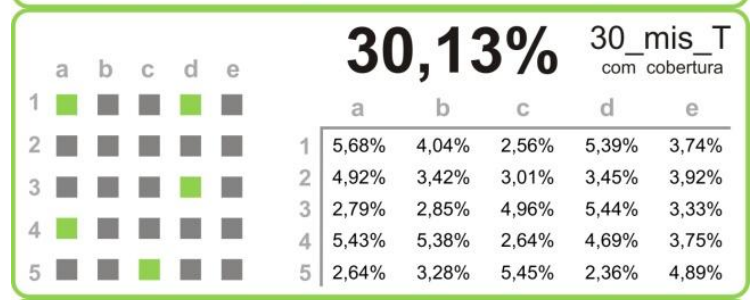

\begin{tabular}{|c|c|c|c|c|c|c|}
\hline a $\quad b \quad c \quad d \quad e$ & & & & & $\begin{array}{r}40 \\
\text { sem }\end{array}$ & nis_T \\
\hline$\square$ & & a & b & c & d & e \\
\hline $2 \square \square$ & 1 & $10,21 \%$ & $4,47 \%$ & $0,08 \%$ & $8,73 \%$ & $2,64 \%$ \\
\hline 3 & 2 & $6,45 \%$ & $1,65 \%$ & $0,87 \%$ & $1,39 \%$ & $3,39 \%$ \\
\hline & 3 & $0,25 \%$ & $0,17 \%$ & $7,33 \%$ & $8,66 \%$ & $1,60 \%$ \\
\hline 4 & 4 & $8,85 \%$ & $8,44 \%$ & $0,00 \%$ & $5,14 \%$ & $2,76 \%$ \\
\hline L & 5 & $0,00 \%$ & $0,92 \%$ & $9,12 \%$ & $0,00 \%$ & $6,88 \%$ \\
\hline
\end{tabular}

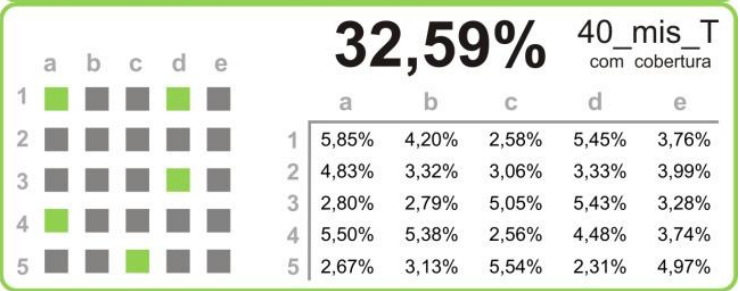


Figura 10 - Fichas-resumo do desempenho particular das edificações e da relação entre geração e consumo dos modelos Miolo de Quadra. Avaliação com e sem cobertura

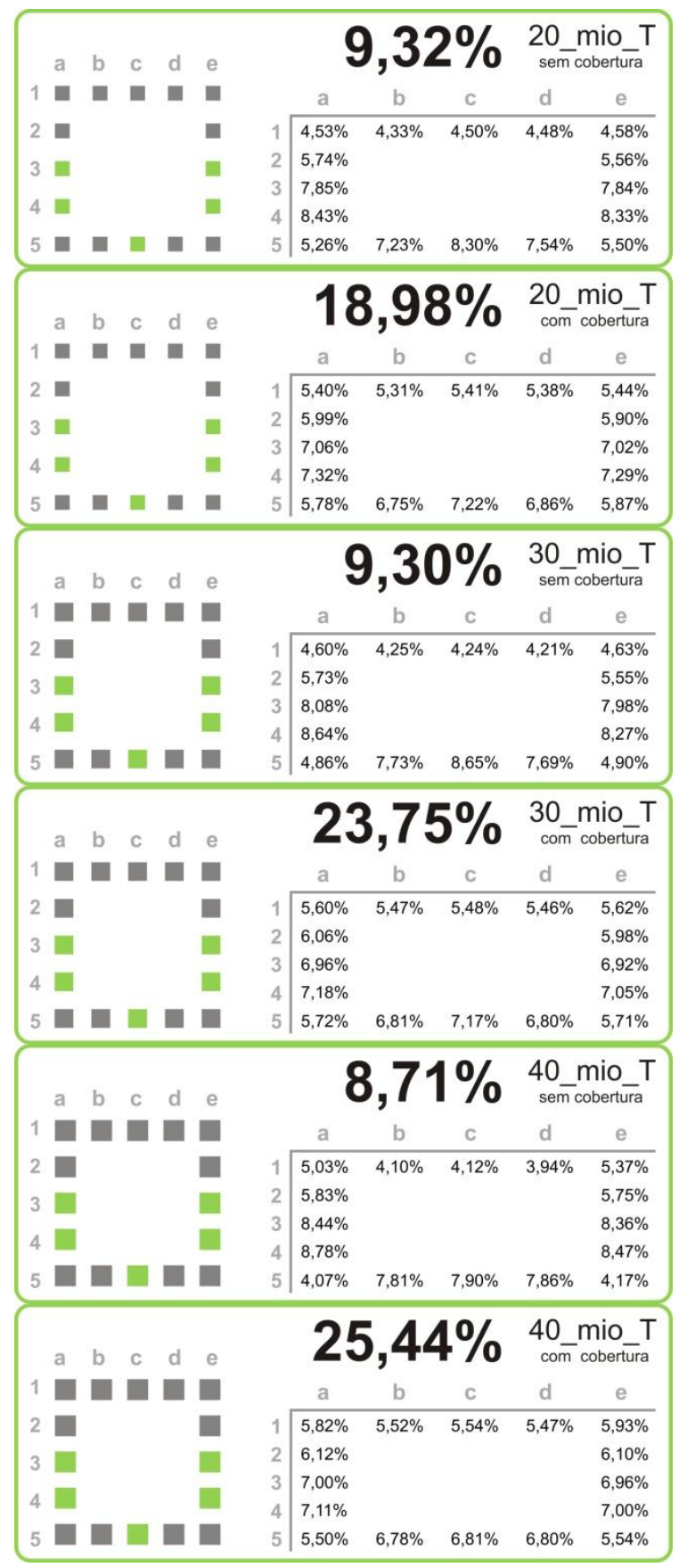

\section{Conclusões}

O desenvolvimento desta pesquisa teve como foco principal a determinação de uma tipologia urbana que apresentasse o melhor potencial de instalação de painéis fotovoltaicos em suas superfícies, tanto verticais quanto horizontais, para o suprimento do consumo anual de energia. Foram definidos também os parâmetros mais influentes na acessibilidade solar dentro dos diferentes contextos urbanos.
Para alcançar o objetivo, foram simulados no plugin DIVA três modelos urbanos com três níveis diferentes de Taxa de Ocupação, e calculada a quantidade de energia que poderia ser gerada em cada cenário, dependendo de sua disponibilidade de radiação solar.

Quando se comparam os resultados compilados dos cenários Mistos e Uniformes, obtêm-se magnitudes bastante similares, indicando que nos modelos Mistos acontece uma compensação entre

20 Gaviria, L. R.; Pereira, F. O. R.; Mizgier, M. O. 
as edificações com muita disponibilidade de radiação solar (mais altos) e os blocos com altos níveis de obstrução solar (mais baixos). Porém, se as edificações são avaliadas separadamente, existem diferenças importantes entre os prédios com os melhores desempenhos em cada modelo. Pode-se assinalar, então, a diferença de altura das edificações como um parâmetro fundamental na acessibilidade solar, quando se analisam os desempenhos das edificações separadamente. Porém, quando avaliado o conjunto, o parâmetro passa a ser menos influente.

A taxa de ocupação também é um parâmetro com bastante peso na determinação da eficiência dos diferentes cenários. Contudo, quando a geração FV é realizada em fachadas e coberturas, o aumento do TO resulta no aumento da eficiência. Já no caso de limitar a integração FV unicamente às fachadas, o aumento do TO é inversamente proporcional aos níveis de eficiência.

Os desempenhos das diversas superfícies das edificações apresentam diferenças importantes, o que indica a orientação como outro parâmetro bastante influente para o cálculo da relação entre geração e consumo.

A influência das coberturas no cálculo da geração energética é bastante importante, porém o aproveitamento das fachadas apresentou bons resultados, principalmente nos modelos com Taxas de Ocupação menores.

É importante levar em consideração que os dados da quantidade de energia gerada por cada cenário são calculados supondo integração FV unicamente nas superfícies opacas. Contudo, o nível de eficiência dos cenários seria sensivelmente incrementado se fosse quantificado também o potencial de integração FV nas superfícies translúcidas. Isso, além do mais, representa uma continuação interessante para a presente pesquisa.

\section{Referências}

\section{AGÊNCIA NACIONAL DE ENERGIA} ELÉTRICA. Resolução Normativa $\mathbf{N}^{\mathbf{0}} \mathbf{4 8 2}$, de 17 de abril de 2012. p. 1-4.

ASSIS, E. S. D. Critérios de Acessibilidade ao Sol e à Luz natural Planejamento Urbano. In: ENCONTRO NACIONAL DE TECNOLOGIA DO AMBIENTE CONSTRUÍDO, 9., Foz do Iguaçu, 2002. Anais... Foz do Iguaçu: ANTAC, 2002.
CHERQUI, F. et al. A Step Toward the Global Assessment of District Projects : solar indicators and way to quantify them. In: INTERNATIONAL IBPSA CONFERENCE, 9., Montreal, 2005. Proceedings...Montreal, p. 167-174, 2005.

CRONEMBERGER, J.; CAAMAÑO-MARTÍN, E.; SÁNCHEZ, S. V. Assessing the Solar Irradiation Potential For Solar Photovoltaic Applications in Buildings at Low Latitudes: making the case for Brazil. Energy and Buildings, v. 55, p. 264-272, set. 2012.

DI VINCENZO, M. C.; KESTEN, D.; INFIELD, D. Assessment of Performance of Building Shading Device With Integrated Photovoltaics in Different Urban Scenarios. In: INTERNATIONAL CONFERENCE ON SUSTAINABLE ENERGY TECHNOLOGIES, Kandy, 2010. Proceedings... Kandy, 2010.

HOFIERKA, J.; KAŇUK, J. Assessment of Photovoltaic Potential in Urban Areas Using Open-Source Solar Radiation Tools. Renewable Energy, v. 34, n. 10, p. 2206-2214, out. 2009.

HWANG, T.; KANG, S.; KIM, J. T. Optimization of the Building Integrated Photovoltaic System in Office Buildings: focus on the orientation, inclined angle and installed area. Energy and Buildings, v. 46, p. 92-104, mar. 2012.

INTERNATIONAL ENERGY AGENCY. Key World Energy Statistics. 2011. Acess em: 15 jun. 2012. Disponível em: <http://www.iea.org/stats/index.asp/>.

JAKUBIEC, J. A.; REINHART, C. DIVA 2.0: integrating daylight and thermal simulations using Rhinoceros 3D, Daysim and Energyplus. In: CONFERENCE OF INTERNATIONAL BUILDING PERFORMANCE SIMULATION ASSOCIATION, 12 ${ }^{\text {th }}$, Sydney, 2011. Proceedings..., Sydney, 2011.

JELLE, B. P.; BREIVIK, C.; RØKENES, H. D. Building Integrated Photovoltaic Products: a stateof-the-art review and future research opportunities. Solar Energy Materials and Solar Cells, v. 100, n. 7465, p. 69-96, maio 2012.

LAMBERTS, R.; PEREIRA, F. O. R.; DUTRA, L. Eficiência Energética na Arquitetura. PRO Livros, São Paulo: $2^{\mathrm{a}}$ ed., 2004.

LEDER, S. M.; PEREIRA, F. O. R. Padrões de Ocupação Urbana e Disponibilidade da Luz Natural. In: ENCONTRO NACIONAL DE CONFORTO NO AMBIENTE CONSTRUÍDO, 10., Natal, 2009. Anais... Natal: ANTAC, 2009. 
LI, D. H. W. et al. A Study of the Daylighting Performance and Energy Use in Heavily Obstructed Residential Buildings Via Computer Simulation Techniques. Energy and Buildings, v. 38, n. 11, p. 1343-1348, nov. 2006.

LITTLEFAIR, P. Passive Solar Urban Design: ensuring the penetration of solar energy into the city. Renewable and Sustainable Energy Reviews, v. 2, n. 3, p. 303-326, set. 1998.

LOULAS, N. M. et al. Photovoltaics in Urban Environment: a case study for typical apartment buildings in Greece. Renewable Energy, v. 48, p. 453-463, dez. 2012.

NG, E.; WONG, N. H. Better Daylight and Natural Ventilation by Design. In: CONFERENCE ON PASSIVE AND LOW ENERGY

ARCHITECTURE, $21^{\text {th }}$, Eindhoven, 2004.

Proceedings... Eindhoven, 2004.

NG, E, Daylighting Simulation of Heavily Obstructed Residential Buildings in Hong Kong. In: INTERNATIONAL BUILDING PERFORMANCE SIMULATION ASSOCIATION CONFERENCE, Rio de Janeiro, 2001. Proceedings... Rio de Janeiro, 2001.

ORDENES, M. et al. The Impact of BuildingIntegrated Photovoltaics on the Energy Demand of Multi-Family Dwellings in Brazil. Energy and Buildings, v. 39, n. 6, p. 629-642, jun. 2007.

PEREIRA, F. O. R. et al. Analysis of Urban Occupation X Daylighting Availability Using the Preferable Sky Window Parameter. In: CONFERENCE ON PASSIVE AND LOW ENERGY ARCHITECTURE, 25 ${ }^{\text {th }}$, Dublin, 2008. Proceedings... Dublin, 2008.

PREFEITURA MUNICIPAL DE FLORIANÓPOLIS; INSTITUTO DE PLANEJAMENTO URBANO DE FLORIANÓPOLIS. Plano Diretor de Florianópolis. 1998.
ROBERT; MCNEEL \& ASSOCIATES. Rhinoceros 4.0 User's Guide. 2008.

RUANO, M. Ecourbanismo: sustainable human settlements: 60 cases studies. Barcelona: [s.n.], 1999.

RÜTHER, R. Edifícios Solares Fotovoltaicos: o potencial da geração solar fotovoltaica integrada a edificações urbanas e interligada à rede elétrica pública no Brasil. USFC/LABSOLAR ed. Florianópolis: 2004.

SANTANA, M. Influência de Parâmetros Construtivos no Consumo de Energia de Edifícios de Escritório Localizados em Florianópolis - SC. Florianópolis, 2006. Dissertação (Mestrado em Engenharia Civil) Departamento da Engenharia Civil, Universidade Federal de Santa Catarina, Florianópolis, 2006.

SOLEMMA. Environmental Tools to Empower Design. Acesso em: 15 abr. 2013. Disponível em: <http://www.solemma.net/>.

SÁNCHEZ DE LA FLOR, F. J. et al. Solar Radiation Calculation Methodology For Building Exterior Surfaces. Solar Energy, v. 79, n. 5, p. 513-522, nov. 2005.

TREGENZA, P. The Design of Lighting. Londres: E\&FN Spon. 1998.

VARTIAINEN, E. Electricity Benefits of Daylighting and Photovoltaics For Various Solar Facade Layouts in Office Buildings. Energy and Buildings, v. 33, n. 2, p. 113-120, 2001.

ZOMER, C. Geração Solar Fotovoltaica Integrada a Uma Edificação Inserida em Meio Urbano e Conectada à Rede Elétrica.

Florianópolis, 2010. Dissertação (Mestrado em Engenharia Civil) - Departamento da Engenharia Civil, Universidade Federal de Santa Catarina, Florianópolis, 2010. 


\section{Laura Rendón Gaviria}

Programa de Pós-graduação em Arquitetura e Urbanismo, Centro Tecnológico | Universidade Federal de Santa Catarina | Campus Universitário, Trindade | Caixa Postal 476 | Florianópolis - SC - Brasil | Tel.: (48) 3721-7080 | E-mail: laura@labcon.ufsc.br

\section{Fernando Oscar Ruttkay Pereria}

Departamento de Arquitetura e Urbanismo, Centro Tecnológico | Universidade Federal de Santa Catarina | E-mail: feco@arq.ufsc.br

\section{Martín Ordenes Mizgier}

Departamento de Arquitetura e Urbanismo, Centro Tecnológico | Universidade Federal de Santa Catarina | E-mail: martin@arq.ufsc.br

\section{Revista Ambiente Construído}

Associação Nacional de Tecnologia do Ambiente Construído

Av. Osvaldo Aranha, $99-3^{\circ}$ andar, Centro

Porto Alegre - RS - Brasil

$$
\text { CEP } 90035-190
$$

Telefone: +55 (51) 3308-4084

Fax: +55 (51) 3308-4054

www.seer.ufrgs.br/ambienteconstruido

E-mail: ambienteconstruido@ufrgs.br 